\title{
El tamaño de las empresas manufactureras y la contaminación medioambiental en México: una aproximación espacial ${ }^{1}$
}

Roldán Andrés-Rosales. Cátedra Conacyt-Centrogeo, Ciudad de México, México. Nam Kwon Mun. Hankuk University of Foreign Studies, Seúl, Corea del Sur. Luis Quintana-Romero. Universidad Nacional Autónoma de México, Ciudad de México, México.

RESUMEN | En este trabajo analizamos la existencia de la Curva de Kuznets Ambiental (CKA) en las distintas regiones mexicanas. Mediante la incorporación de las empresas manufactureras según su tamaño y los distintos niveles de contaminación ambiental, brindamos evidencia sobre el cumplimiento de dicha curva para ciertas regiones del país. Con tal fin, hacemos uso de la información oficial disponible para el periodo 1999-2009 y mediante un modelo econométrico de panel espacial mostramos que, en las regiones con predominio de micro y pequeńas empresas, no se cumple la CKA. A pesar de que son abundantes los estudios sobre la CKA, no se había puesto suficiente atención al papel que desempeńa la estructura productiva de las empresas locales en el medioambiente.

PALABRAS CLAVE | indicadores ambientales, distribución espacial, desarrollo regional y local.

ABSTRACT | In this paper we analyze the existence of the Environmental Kuznets Curve $(E K C)$ in the different Mexican regions. By incorporating the manufacturing companies according to their size and the different levels of environmental pollution, we provide evidence of the compliance with the curve for certain regions of the country. For this, we use official data available for the period 1999-2009 to formulate and evaluate a spatial panel econometric model, which shows that the $E K C$ is not met in the regions where micro and small enterprises are predominant. Although there are several studies regarding the $E K C$, the role of the productive structure of local companies on the environment has not been analyzed deeply.

KEYWORDs | environmental indicators, spatial distribution, regional and local development.

1 Este trabajo se realizó con el apoyo del Fondo de Investigación de la Universidad Hankuk de Estudios Extranjeros (Hankuk University of Foreign Studies Research Fund) de 2017.

This work was supported by the National Research Foundation of Korea Grant funded by the Korean Government(NRF-2012S1A5B8A03045253) 


\section{Introducción}

Con la firma del Tratado de Libre Comercio de América del Norte (TLCAN) se abrió un amplio debate sobre la posible agudización de la contaminación medioambiental por la apertura comercial. Al centro de la discusión estaba la incertidumbre sobre los efectos adversos que la mayor relación comercial entre México y Estados Unidos provocaría en el medioambiente de ambos países (Gallagher, 1999). Dentro del debate, una de las posiciones más críticas era la de grupos medioambientalistas y sindicatos que sustentaba la hipótesis del paraíso contaminante (pollution haven hypothesis, $\mathrm{PHH})$; su argumento central era que la expansión del libre comercio provocaría la destrucción medioambiental de México, ya que por ser un país de ingresos bajos y contar con débiles instituciones regulatorias, resultaría altamente atractivo para que las empresas más contaminantes se relocalizaran en su territorio (Gallagher, 2004). El temor de los críticos era que, dadas las normas ambientales mexicanas poco estrictas, la intensificación de la producción y del comercio provocaría más contaminación y esta traspasaría la frontera desde Estados Unidos (Comisión Económica para América Latina y el Caribe [CEPAL], 2004).

En contraposición a la PHH, autores como Gallagher (1999, 2004), Harbaugh, Levinson y Wilson (2002), Boopen y Vinesh, (2010), argumentaban una visión contraria y menos pesimista sustentada en la existencia de una curva de Kuznets ambiental (CKA). Para ellos, el libre comercio se convertiría en un factor positivo para el medioambiente, porque impactaría favorablemente en la producción del país, y las empresas transnacionales que se instalaran en México, al contar con tecnología de punta, disminuirían la emisión de contaminantes. El sustento económico de esta visión más optimista se encuentra en la idea de que la calidad medioambiental es un bien de lujo, lo cual implica que su elasticidad de demanda varía con el nivel de ingreso (Yandle, Bhattarai \& Vijayaghavan, 2004).

Si bien la discusión sobre la existencia de la CKA se ha documentado en numerosos artículos, existe poca evidencia de sus implicaciones regionales y sobre el papel específico que el tamaño de la empresa manufacturera ha desempeñado en la contaminación. Por ello, en este trabajo se pretende explorar la hipótesis de la CKA en los estados mexicanos y mostrar si el tamaño de empresa es relevante para la existencia de dicha curva. Esta hipótesis es relevante en la medida en que se ha considerado que con la firma y entrada en vigor del TLCAN ha habido una restructuración productiva en México, que ha favorecido relativamente más a la expansión de las grandes empresas (López-Córdova, 2001). Esta dinámica productiva diferencial hace posible suponer que el tamaño de las empresas puede tener efectos diferenciados en las emisiones contaminantes en las entidades del país y que, donde se han concentrado las grandes empresas, podría haberse favorecido el cuidado medioambiental, como lo plantea la CKA.

El presente trabajo aborda, en la segunda sección, la evidencia empírica y teórica sobre las hipótesis de la PHH y de la CKA. En la tercera parte se estudia la estructura productiva de las regiones mexicanas según el tamaño de las empresas manufactureras y su papel en la generación de contaminantes en los estados mexicanos. A continuación, se especifica un modelo econométrico empírico consistente con la 
existencia de la CKA y se analizan sus resultados. Finalmente, en la última sección se establecen las principales conclusiones del trabajo.

\section{Análisis de la hipótesis del paraíso contaminante (PPH) y la curva de Kuznets ambiental (CKA)}

Durante los noventa, el debate sobre el efecto del libre comercio en el medioambiente de México se agudizó. Los argumentos eran contrastantes: uno de ellos sostenía que este país se convertiría en un paraíso contaminante debido a la destrucción medioambiental acelerada; el otro, en tanto, argumentaba la mejora ambiental debido al aumento del ingreso provocado por el crecimiento económico resultante de la apertura comercial (Boopen \& Vinesh, 2010; Cole, 2003; Gallagher, 1999, 2004; Harbaugh, Levinson \& Wilson, 2002; Stern \& Common, 2001). Se afirmaba que empresas transnacionales altamente competitivas se establecerían en México, por las ventajas de menores costos salariales y una ubicación geográfica privilegiada respecto de los Estados Unidos. La curva de Kuznets, formulada originalmente para analizar la relación entre ingreso y desigualdad, se trasladó al estudio del comercio y medioambiente (Gallagher, 1999, 2004). Dentro de esta discusión, Grossman y Kruger (1991) estudiaron el impacto medioambiental de la liberalización comercial bajo tres efectos: efecto escala, efecto tecnológico y efecto estructural. El efecto escala, asociado a un mayor acceso al mercado, se esperaba provocaría una mayor contaminación debido la existencia de crecimiento económico, pero sin cambio tecnológico. En tanto que los efectos tecnológico y estructural, al impactar positivamente la productividad y el ingreso, fortalecerían la regulación medioambiental y estimularían la disminución en la emisión de contaminantes (Cole, 2003; Panayotou, Peterson \& Sachs, 2000; Stern, 1998, 2004; Stern, Common \& Barbier, 1996; Suri \& Chapman, 1998).

Buena parte de la literatura empírica de la CKA se ha dedicado a identificar el umbral de ingreso a partir del cual la contaminación tendería a declinar; al respecto, Grossman y Krueger (1991) brindaron una de las primeras evidencias, al señalar que cuando un país lograba un ingreso per cápita de 5.000 dólares (a precios de 1985), se encontraba en ese umbral. Sin embargo, la abundante literatura que siguió a ese trabajo seminal no ha dado lugar a resultados concluyentes (Birdsall \& Wheeler, 1993; Day \& Grafton, 2001; Jones \& Manuelli, 2000; López, 1994; Roca \& Padilla, 2003). Hay indefinición sobre el umbral de ingreso, que incluso ha fluctuado entre los 7.500 y los 15.000 dólares (Gallagher, 2006). Pero también hay quienes cuestionan la existencia de tal umbral y defienden diferentes puntos de quiebre dependiendo del tipo de contaminante del que se trate (Selden \& Song, 1994). Lo relevante de esta discusión es que, debido a que la existencia de la CKA depende del tipo específico de contaminante del que se hable, no es posible estudiar el problema de manera agregada (Lieb, 2003).

Para el caso particular de México, la evidencia empírica sigue siendo escasa (Gallagher, 2004; Saravia, 2002; Seldeng \& Song, 1994; Stern, 2004; Tarazona, 1999) y la existente se ha concentrado en torno a los impactos medioambientales del TLCan. Estos estudios, según Carson (2010), han mostrado que el incremento 
en el ingreso no se asocia automáticamente con el incremento de la contaminación; que el libre comercio no ocasiona necesariamente el empeoramiento del deterioro ambiental; y que el TLCAN pudo haber ocasionado una mejora ambiental. Incluso se ha mostrado que las empresas contaminantes se han incrementado en los Estados Unidos y no en México, con lo que podría deducirse que estas empresas no han migrado y considerado a México como un paraíso contaminante (Gallagher, 2000). También Antwiler, Copeland y Taylor (2001) y Ederington, Levinson y Minier (2004) presentaron resultados opuestos a la hipótesis planteada por la PHH en el sentido de que Estados Unidos registra mayor densidad contaminante en sus exportaciones que en sus importaciones. Stern (2004) analizó los datos de emisión de contaminantes y uso de energía para el período 1971-2003 cuando México cambió del proteccionismo al librecambismo, y concluye que no se detectó cambio alguno en la tendencia de emisión contaminante en los tres países. Uno de los estudios más recientes muestra que el crecimiento económico en México después del TLCAN ha sido insuficiente para dar lugar a una amplia mejora medioambiental, en particular consideran que el ritmo de crecimiento del PIB per cápita es muy bajo y no ha permitido generar un cambio estructural relevante en el país (Lipford \& Yandle, 2011).

En las discusiones anteriores, el tamaño de la empresa ha sido ignorada por la literatura (Merlevedea, Verbekeb \& Clercq, 2006), por lo que existe poca evidencia empírica sobre su relevancia en la generación de emisiones contaminantes. En los estudios que se han hecho sobre el tema, los resultados varían dependiendo del tipo de país; por ejemplo, para las pequeñas empresas mexicanas y brasileńas hay trabajos que muestran que son más contaminantes por hombre-ocupado, aunque las grandes empresas siguen contabilizando las mayores cantidades de emisiones en el agregado (Dasgupta, Lucas \& Wheeler, 2002). Para el Reino Unido se encontró que la relación entre emisiones contaminantes y tamańo de empresa era negativa (Cole, Elliott \& Shimamoto, 2005). En la relación entre tamaño de empresa y contaminación, el caso chino es el que más se ha estudiado: la evidencia para ese país indica que las grandes empresas emiten menos contaminantes por unidad de producto, y que ello se debe al uso de tecnologías de producción ambientalmente más amigables y tecnologías de tratamiento de contaminantes más avanzadas (Qi, Tang \& Xi, 2015). También se ha encontrado que, en China, las pequeñas empresas descargan más contaminantes que las grandes empresas y, sin embargo, el gobierno es indulgente con ellas debido a que son proveedoras de empleo, lo cual tiende a agravar el problema de la contaminación (Jiang, Chen \& Ping, 2014). También se ha considerado que, en general, las pequeñas y medianas empresas (PYMEs) suelen ser responsables de aproximadamente el $60 \%$ de las emisiones de dióxido de carbono y del $70 \%$ de la contaminación, debido a que tienen recursos limitados para operar y no cuentan con las capacidades tecnológicas para atenuar el impacto ambiental (Marshall, 1998; Parker, Redmond \& Simpson, 2009; Revell \& Blackburn, 2007; Revell, Stokes \& Chen, 2010).

En general, la evidencia respecto a México tiene tres serias limitaciones. La primera es que, en su mayor parte, la contaminación ambiental originada en la actividad productiva se generó cuando México apenas estaba insertándose al TLCAN; por consiguiente, hay escases de trabajos más recientes que busquen investigar si 
las tendencias ya detectadas se mantienen. La segunda limitante consiste en que prácticamente toda la evidencia es agregada, dando por hecho la existencia de homogeneidad productiva en todas las regiones del país. La tercera tiene que ver con el hecho de que la literatura sobre el país no ha indagado en el papel regional diferencial que tiene la manufactura en el deterioro medioambiental.

La discusión previa y, en particular, la indefinición sobre los efectos del tamaño de empresa en la contaminación, justifican profundizar en el estudio de este tema, lo cual se efectuará en las siguientes secciones condicionado a diferentes tipos de contaminantes y a diferentes entidades federativas del país.

\section{Distribución espacial de las empresas manufactureras en las regiones mexicanas y la contaminación ambiental}

En México, las pymes representan una parte fundamental de la economía: de acuerdo con los datos del último Censo Económico de 2009, del total de unidades económicas del país, el 95,2\% son microempresas, el 4,3\% pequeñas empresas, un $0,3 \%$ son medianas y solo el 0,25 son empresas grandes. En consecuencia, el 78,5\% del empleo en el país es generado por las pyMEs.

En la figura 1 se muestran algunas comparaciones entre las emisiones en toneladas por persona de diferentes tipos de contaminantes y el valor agregado por tamaño de empresa para 1999 y 2009. El primer mapa corresponde al monóxido de carbono (CO); el color negro indica elevadas emisiones, destacando en 1999 los estados de Nuevo León, Tamaulipas, Distrito Federal, Campeche, Chiapas, Oaxaca y Guerrero. Para 2009 son otros los estados con mayores emisiones de co: Baja California, Coahuila, Zacatecas, Jalisco, Michoacán y Morelos. Con respecto al dióxido de sulfuro $\left(\mathrm{SO}_{2}\right)$, en ambos ańos sobresalen los estados de Coahuila, Hidalgo, Colima, Guerrero y Campeche; mientras que en óxido de nitrógeno (Nox) destacan Coahuila, Tamaulipas, Colima y Campeche. En partículas menores a 10 micrómetros (PMIO), las mayores emisiones corresponden a Nayarit, Guerrero, Oaxaca, Campeche y Quintana Roo. La figura 1C muestra el valor agregado de las microempresas (Micro), pequeñas y medianas empresas (PYMEs) y de las grandes empresas $(\mathrm{Ge})$. Las Ge se concentran en las entidades de Baja California, Chihuahua, Coahuila, Nuevo León, Aguascalientes y Querétaro en ambos años, en tanto que las pymes lo hacen en Baja California, Coahuila, Nuevo León, Jalisco y Tlaxcala; mientras que las Micro se encuentran en mayor proporción en Baja California Sur, Nuevo León, Jalisco y Distrito Federal. 

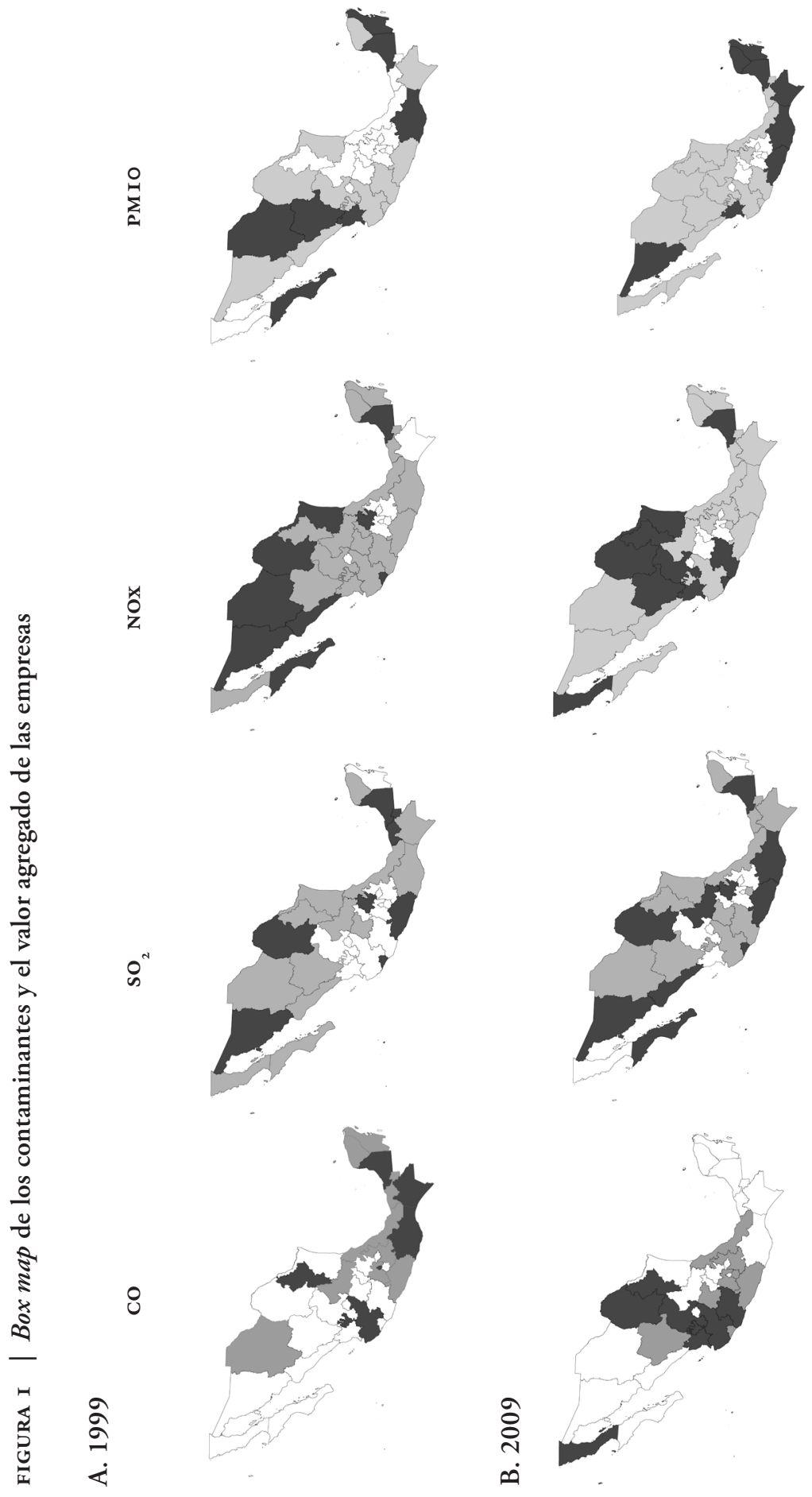


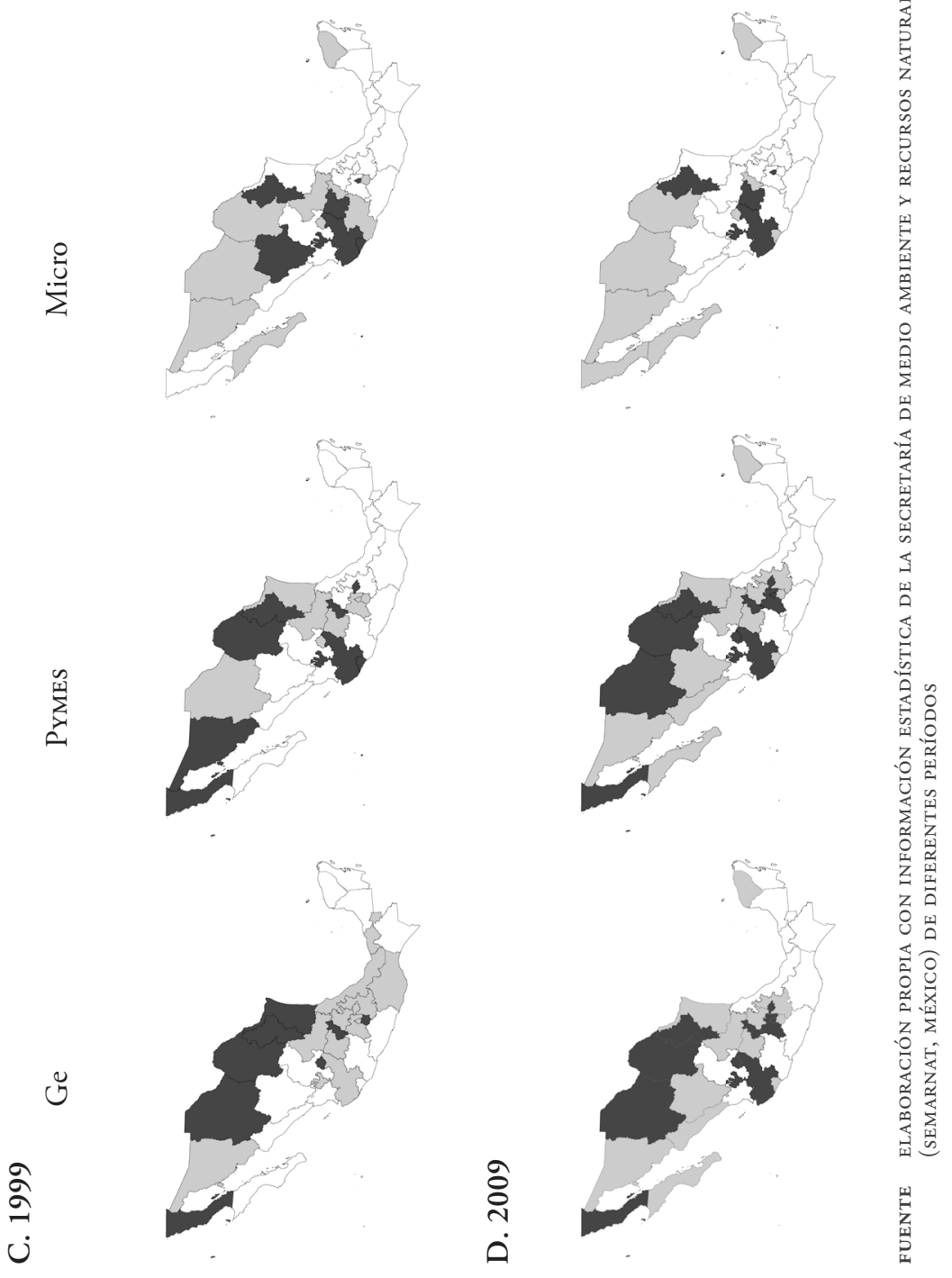




\section{Planteamiento del modelo}

De acuerdo con los modelos empíricos que se han utilizado para estimar ecuaciones de la CKA, es usual tomar como variable dependiente las emisiones per cápita de algún tipo de contaminante; y como variables independientes, la inversión, PIB, educación, vehículos, población ocupada, entre otras (Boopen \& Vinesh, 2010; Díaz-Vázquez, 2009; Díaz-Vázquez \& Cancelo, 2009; Gallagher, 1999; Roca \& Padilla, 2003). En la ecuación (1) se muestra el modelo estándar de panel utilizado en este tipo de trabajos (Dinda, 2004):

$$
y_{i t}=\alpha_{i}+\beta_{1} x_{i t}+\beta_{2} x_{i t}^{2}+\beta_{3} x_{i t}^{3}+\beta_{4} z_{i t}+\varepsilon_{i t}
$$

donde $\mathrm{y}_{\mathrm{it}}$ es un indicador medioambiental, $\mathrm{x}_{\mathrm{it}}$ es el ingreso $\mathrm{y}_{\mathrm{it}}$ es un indicador de otras variables que degradan el ambiente.

El caso particular de la CKA se presenta cuando $\beta_{1}>0, \beta_{2}<0$ y $\beta_{3}=0$

Esta primera ecuación se utilizó en los primeros planteamientos de la CKA, que critica Stern (2004); de ahí que expandamos el planteamiento anterior agregando otras variables que puedan influir en la contaminación. Es decir, los contaminantes como el monóxido de carbono (CO), el óxido de nitrógeno (NOx) y partículas finas (PMIO) tienen diferentes fuentes de emisión. Por ejemplo, el primero se produce en la gasolina, calentadores de agua, equipos de motor, el petróleo y la leña, entre otros. El Nox tiene un origen similar, sumando además la combustión del carbón, petróleo o gas natural. Por su parte, el dióxido de azufre $\left(\mathrm{SO}_{2}\right)$ es generalmente causado por las industrias y plantas de generación eléctrica, y substancias químicas que se utilizan en gran cantidad en la industria, en sistemas de refrigeración, aire acondicionado y en la elaboración de bienes de consumo (Hertwich, 2011). De ahí que estos contaminantes sean considerados en la variable dependiente de estos modelos.

En este trabajo se propone una especificación que es una variante de la ecuación (1) para incorporar el tamaño de empresa en el modelo. La especificación se muestra en la ecuación (2):

$$
\begin{aligned}
& p o_{i t, j}=\alpha_{i}+\rho W p o_{i t}+\beta_{1} v a_{i t, j}+\beta_{2} v a_{i t, j}^{2}+\beta_{3} k_{i t, j}+\beta_{4} e u_{i t, j}+e_{i t, j} \\
& e_{i t, j}=\lambda W e_{i t, j}+v_{i t, j}
\end{aligned}
$$

donde $\mathrm{po}_{\mathrm{it}}$ son los contaminantes ( $\mathrm{CO}, \mathrm{SO}_{2}$, NOx, y PMIO) divididos por la población total de la entidad; $\mathrm{va}_{\mathrm{it}}$ es el valor agregado per cápita; $\mathrm{k}_{\mathrm{it}}$ son los acervos brutos de capital fijo por unidad de producto; y eu $\mathrm{i}_{\mathrm{it}}$ es el porcentaje del número de empresas (unidades económicas) por tamaño (micro, pequeña, mediana y grande) en cada entidad federativa. Todas las variables se transformaron en logaritmos y se indexan para cada entidad federativa i, en el ańo t y para el tamańo de empresa $j$ (micro, pequeña, mediana y grande).

Con la ecuación (2) se pretende incorporar el efecto del tamaño de la empresa manufacturera sobre el incremento de los contaminantes mencionados; esto se realiza tanto por el efecto productivo de estas empresas (valor agregado), como por su número en la economía (unidades económicas). Además se incluye el capital per cápita como una variable proxy del progreso tecnológico. De esta forma, la ecuación (1) también es modificada en la (2) para ser estimada mediante técnicas de panel 
espacial (Anselin, 1988; Anselin \& Florax, 1995; Arbia, 2008; Cliff \& Ord, 1972; Paelinck \& Klaassen, 1979). Con ese fin se ha incorporado una estructura de dependencia espacial para evaluar la posible existencia de efectos de derrame o spillovers en la contaminación entre los estados mexicanos. La incorporación de la dependencia se realiza a través de un término de rezago espacial en la ecuación $\left(\rho W p o_{i t}\right)$ y en la estructura del término de perturbación del modelo $\left(\lambda W e_{i t, j}\right)$, siendo $\mathrm{W}$ una matriz de pesos espaciales cuyos elementos toman el valor de 1 cuando la unidad territorial presenta vecindades y 0 en otros casos.

Basados en la ecuación (2), podemos tener dos casos particulares: el primero cuando $\lambda=0$ y $\rho \neq 0$ es el modelo de rezago espacial. El segundo, cuando $\lambda \neq 0$ y $\rho=0$ es el modelo de error espacial. La selección de estos modelos se realiza utilizando las pruebas LM y sus versiones robustas propuestas por Anselin (1988).

La ecuación (2) es estimada por datos de panel espacial para lograr incorporar la heterogeneidad geográfica de la información y capturar la interacción espacial entre las unidades económicas a través del tiempo (Millo \& Piras, 2012). La heterogeneidad espacial entre los estados mexicanos es tratada a través de la incorporación del efecto fijo $\left(\alpha_{i}\right)\left(\alpha_{i}\right)$ (Anselin, LeGayo \& Jayet, 2008; Elhorst, 2003, 2010). Algunos de los problemas que considera Stern (2004) sobre las técnicas econométricas usadas en el estudio de CKA son: dependencia serial o tendencias estocásticas en series de tiempo, heteroscedasticidad, simultaneidad, omisión de variables relevantes y problemas de cointegración. De ahí que en este trabajo se utilice la técnica de datos de panel espacial, cuyo principal objetivo es capturar la heterogeneidad no observable, que puede afectar la contaminación medioambiental. Dado que la heterogeneidad no se puede detectar ni con estudios de series temporales ni con los de corte transversal, la técnica de panel permite realizar un análisis más dinámico al incorporar la dimensión temporal de los datos, lo que enriquece el estudio, en especial en periodos de grandes cambios (Wooldridge, 2002). Los datos de panel proporcionan más información, más variabilidad y menos colinealidad entre las variables, más grados de libertad y mayor eficiencia. Las series de tiempo están plagadas de multicolinealidad (Baltagi, 2005).

Sumado a lo anterior, Lesage y Fisher (2008) y Lesage y Pace (2009) examinan las ventajas de usar el modelo espacial de Durbin (MDs). Consideran que en el análisis regional se ha incrementado el uso de estos modelos porque permiten capturar la dependencia espacial en todos los regresores (Cravo, Gourlay \& Becker, 2012), externalidades espaciales y efectos de derrame o spillovers (Ertur \& Koch, 2007; López-Bazo, Vaya \& Artis, 2004). Debido a ello, en este trabajo se utiliza también una especificación MDs de la ecuación (2), en la cual se introduce un rezago espacial para cada una de las variables explicativas del modelo. El modelo espacial general MDS se presenta en la ecuación (3):

$$
\begin{aligned}
& p o_{i t, j}=\alpha_{i}+\rho W p o_{i t}+\beta_{1} v a_{i t, j}+\beta_{2} v a_{i t, j}^{2}+\beta_{3} k_{i t, j}+\beta_{4} e u_{i t, j}+\tau_{1} W v a_{i t, j}+\tau_{2} W v a_{i t, j}^{2}+ \\
& \tau_{3} W k_{i t, j}+\tau_{4} W e u_{i t, j}+e_{i t, j} \\
& e_{i t, j}=\lambda W e_{i t, j}+v_{i t, j}
\end{aligned}
$$


Así, tendremos un modelo Durbin de rezago espacial cuando $\lambda=0, \rho \neq 0$ y $\tau_{k} \neq 0$ siendo $\mathrm{k}=1,2,3$, mientras que cuando $\lambda \neq 0, \rho=0$ y $\tau_{k} \neq 0$ será un modelo Durbin de error espacial. En el caso de que $\lambda=0, \rho=0$ y $\tau_{k} \neq$ tendremos un modelo de rezago espacial en los regresores o sLX.

\section{Datos}

Para la estimación de las diferentes variantes de la ecuación (3) se utilizó información proveniente de la Secretaría de Medio Ambiente y Recursos Naturales (Semarnat), institución que mantiene un monitoreo constante de la contaminación medioambiental de los diferentes estados mexicanos, principalmente de su zona metropolitana. Las variables económicas y de las empresas manufactureras provienen de los Censos Económicos (Instituto Nacional de Estadística y Geografía [Inegi]) de diferentes periodos. La contaminación ambiental se encuentra expresada en toneladas, las que se transformaron en términos per cápita y logarítmicas. La clasificación de las PYMEs se realiza con la metodología proporcionada por el Inegi y se restringe al sector manufacturero; las variables se encuentran expresadas en términos reales a precios de 2003. Finalmente, dividimos las empresas en cuatro grupos, usando la población ocupada total: micro: $\leq 10$, pequeña: $11-50$, mediana: $51-250$, y grande: 2251. La información proviene de los Censos Económicos de 1999, 2004 y 2009. El periodo de la información es censal, lo que imposibilita obtener periodos largos. Para subsanar esta deficiencia usamos el estimador de datos de panel, que permite obtener mejores estimaciones cuando trabajamos con datos de sección cruzada y corte transversal, e incrementa el número de observaciones.

\section{Evidencia empírica de la contaminación medioambiental en México}

En la tabla 1 se presentan los resultados de la estimación de la ecuación (3) para el caso de las microempresas y considerando solo aquellos contaminantes en los que resultó significativo el modelo. De la tabla se desprende que para el modelo puro de CKA (columna A de la tabla 1), el valor agregado de las microempresas tiene un impacto negativo en las emisiones de CO (-7,179 para el valor agregado y -0,526 para su cuadrado). Sin embargo, en las columnas (B), (C) y (D) se ha condicionado la ecuación incorporando como regresores al capital, al peso de las unidades económicas y al conjunto de dichas variables, respectivamente. De esas estimaciones se concluye que la única variable relevante es el número proporcional de unidades económicas, lo cual podría reflejar el hecho de que las microempresas son grandes generadoras de empleo, pero no lo son en la producción. Por ello, su impacto en la generación de contaminantes podría estar más asociado al número de empresas que al nivel de producción que generan. En las columnas (E) a la (H) se aprecian los resultados de las especificaciones MDs. En este caso resultan significativos los rezagos espaciales sobre el valor agregado y su cuadrado, lo cual indicaría que en un estado se reducirían las emisiones contaminantes cuando se eleva la producción de las microempresas en los estados vecinos. Esto último podría ser la consecuencia de un efecto espacial tipo 
centro-periferia, en el cual, al crecer las microempresas en una entidad, tenderían a desestimular la producción en sus vecindades. En cualquiera de los casos, la evidencia obtenida no resulta consistente con el cumplimiento de la hipótesis CKA.

TABLA I | Estimación de los contaminantes y las microempresas con datos de panel y modelo Durbin, 1999-2009

\begin{tabular}{|c|c|c|c|c|c|c|c|c|}
\hline \multirow{2}{*}{ MICRO } & \multicolumn{8}{|c|}{ I) MONÓXIDO DE CARBONO (CO) } \\
\hline & \multicolumn{4}{|c|}{ DATOS DE PANEL } & \multicolumn{4}{|c|}{ MODELO DURBIN } \\
\hline \multirow[b]{2}{*}{ VARIABLES } & A) & B) & c) & D) & E) & F) & G) & н) \\
\hline & ORIGINAL & CAPITAL & $\begin{array}{c}\text { UNIDADES } \\
\text { ECONÓ- } \\
\text { MICAS }\end{array}$ & TOTAL & ORIGINAL & CAPITAL & $\begin{array}{c}\text { UNIDADES } \\
\text { ECONÓ- } \\
\text { MICAS }\end{array}$ & TOTAL \\
\hline \multirow{2}{*}{ va } & $-7,179^{* * *}$ & $-3,421$ & $-0,582$ & $-0,441$ & 3,662 & $5,1459 *$ & 3,979 & 3,922 \\
\hline & $(-2,971)$ & $(-1,429)$ & $(-0,308)$ & $(-0,228)$ & $(1,155)$ & $(1,821)$ & $(1,622)$ & $(1,542)$ \\
\hline \multirow{2}{*}{ va2 } & $-0,526^{* * *}$ & $-0,273$ & $-0,073$ & $-0,063$ & 0,291 & $0,382 *$ & 0,296 & 0,292 \\
\hline & $(-2,834)$ & $(-1,515)$ & $(-0,513)$ & $(-0,44)$ & $(1,225)$ & $(1,808)$ & $(1,604)$ & $(1,528)$ \\
\hline \multirow{2}{*}{$\mathrm{k}$} & & $-0,782 * * *$ & & $-0,077$ & & $-0,246$ & & $-0,042$ \\
\hline & & $(-3,845)$ & & $(-0,398)$ & & $(-1,155)$ & & $(0,198)$ \\
\hline \multirow{2}{*}{ ue } & & & $2,019^{* * *}$ & $1,948^{* * *}$ & & & 2,224 & 1,875 \\
\hline & & & $(8,021)$ & $(6,284)$ & & & $(0,632)$ & $(0,474)$ \\
\hline \multirow[t]{2}{*}{ constante } & $-25,901^{\text {*ak }}$ & $-12,749$ & $-1,973$ & $-1,51$ & $-57,95^{* * *}$ & $-32,163^{* * *}$ & $-23,45^{* *}$ & $-23,219^{* *}$ \\
\hline & $(-3,329)$ & $(-1,629)$ & $(0,316)$ & $(-0,236)$ & $(-5,866)$ & $(-3,043)$ & $(-2,531)$ & $(-2,403)$ \\
\hline \multirow{2}{*}{$\mathrm{w}^{*} \mathrm{va}$} & & & & & $-20,79^{* * *}$ & $-14,77^{* * *}$ & $-11,124^{* * *}$ & $-10,995^{* k *}$ \\
\hline & & & & & $(-4,674)$ & $(-3,53)$ & $(-2,965)$ & $(-2,843)$ \\
\hline \multirow{2}{*}{$\mathrm{w}^{*} \mathrm{va2}$} & & & & & $-1,582$ *** & $-1,149^{* * *}$ & $-0,866^{* * *}$ & $-0,857^{* * *}$ \\
\hline & & & & & $(-4,613)$ & $(-3,593)$ & $(-3,005)$ & $(-2,885)$ \\
\hline \multirow{2}{*}{$\mathrm{w}^{*} \mathrm{k}$} & & & & & & $-0,864 * * *$ & & 0,042 \\
\hline & & & & & & $(-3,058)$ & & $(0,125)$ \\
\hline \multirow[t]{2}{*}{$w^{*}$ ue } & & & & & & & $-0,497$ & $-0,147$ \\
\hline & & & & & & & $(0,142)$ & $(-0,037)$ \\
\hline
\end{tabular}

\begin{tabular}{|c|c|c|c|c|c|c|c|c|}
\hline & \multicolumn{8}{|c|}{ II) PARTÍCULAS FINAS (PMIO) } \\
\hline & \multicolumn{4}{|c|}{ DATOS DE PANEL } & \multicolumn{4}{|c|}{ MODELO DURBIN } \\
\hline \multirow[b]{2}{*}{ VARIABLES } & A) & в) & c) & D) & E) & F) & G) & H) \\
\hline & ORIGINAL & CAPITAL & $\begin{array}{c}\text { UNIDADES } \\
\text { ECONÓ- } \\
\text { MICAS }\end{array}$ & TOTAL & ORIGINAL & CAPITAL & \begin{tabular}{|c|} 
UNIDADES \\
ECONÓ- \\
MICAS
\end{tabular} & TOTAL \\
\hline \multirow{2}{*}{ va } & $2,594^{* *}$ & $3,515^{* * *}$ & $3,334^{* * *}$ & $3,631^{* * *}$ & $2,708^{*}$ & $2,769^{*}$ & $3,023^{* *}$ & $2,818^{* *}$ \\
\hline & $(2,645)$ & $(3,369)$ & $(3,097)$ & $(3,351)$ & $(1,889)$ & $(1,95)$ & $(2,218)$ & $(2,01)$ \\
\hline \multirow{2}{*}{ va2 } & $0,182^{* *}$ & $0,244^{* * *}$ & $0,232 * * *$ & $0,252^{* * *}$ & 0,147 & 0,152 & $0,178^{*}$ & 0,163 \\
\hline & $(2,413)$ & $(3,102)$ & $(2,867)$ & $(3,099)$ & $(1,367)$ & $(1,436)$ & $(1,735)$ & $(1,551)$ \\
\hline \multirow{2}{*}{$\mathrm{k}$} & & $-0,191^{* *}$ & & $-0,164$ & & $-0,209^{*}$ & & $-0,089$ \\
\hline & & $(-2,163)$ & & $(-1,506)$ & & $(-1,951)$ & & $(-0,763)$ \\
\hline
\end{tabular}


(continuación)

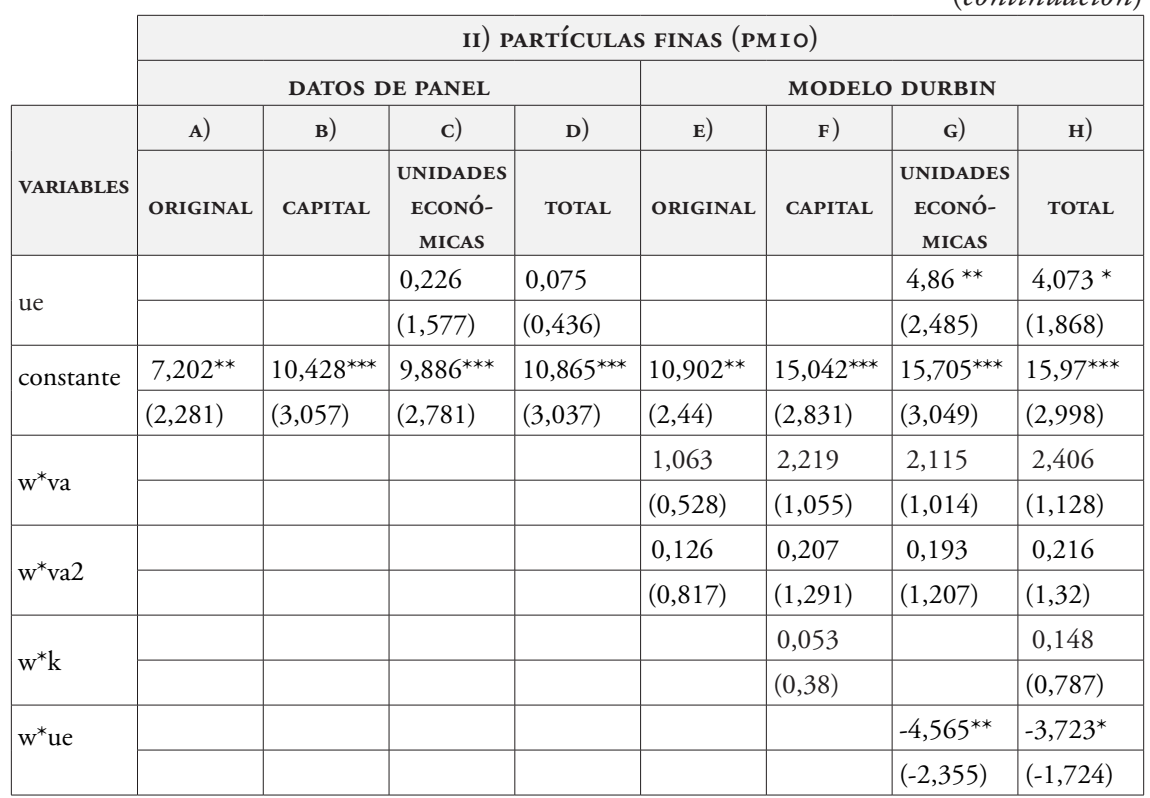

FUENTE ELABORACIÓN PROPIA CON BASE EN INFORMACIÓN DE LOS CENSOS ECONÓMICOS Y DEL SEMARNAT DE I 999, 2004 Y 2009. ${ }^{* * *},{ }^{* *}, *$ : SON LOS NIVELES DE SIGNIFICANCIA DEL I \%, $5 \%$ Y IO\%. EN LOS PARÉNTESIS SE MUESTRAN LOS ESTADÍSTICOS T DE CADA COEFICIENTE ESTIMADO

En la misma tabla 1 se muestran los resultados para las pMio en microempresas. En la columna a el modelo tradicional de CKA indica impactos positivos y significativos del valor agregado y su cuadrado (coeficientes de 2,594 y 0,182 ). Lo mismo ocurre en los modelos alternativos (columnas B, c y D), lo cual permite conjeturar el rechazo a la existencia de una CKA. En este caso, la estimación del modelo espacial Durbin (columnas E, F, G, H) permite observar la existencia de efectos espaciales solo en el caso de las unidades económicas vecinas, y ellos son negativos. Esto supondría que las emisiones se reducen en un estado al crecer el número de empresas de sus vecinos, ello nuevamente como un efecto centro-periferia al que ya se hizo referencia.

En la tabla 2 se presentan los resultados para las PyMEs, omitiendo las ecuaciones para el monóxido de carbono ( $\mathrm{CO}$ ) y el óxido de nitrógeno (NOx), debido a que no resultaron significativas. En cuanto al dióxido de azufre, no es posible confirmar alguna evidencia de una curva de Kuznets, ello en la medida en que tanto el valor agregado como su cuadrado tienen impactos positivos y significativos en la generación de emisiones (columnas A, B, C y D). La inclusión de efectos espaciales en el modelo Durbin no resulta significativa (columnas E, F, G y H) y solo confirma los resultados ya obtenidos en el modelo no espacial.

La estimación de la ecuación (1) para la emisión de pM io en las PYMEs no muestra algún tipo de significancia para las variables incluidas (columnas A, B, C y D) y no permite confirmar la existencia de una CKA; sin embargo, en el modelo Durbin espacial sí resultan relevantes y positivos el crecimiento de la producción y su cuadrado 
en las entidades vecinas. Esto último significa que para las PyMEs hay algún tipo de efecto indirecto que incrementa en una entidad federativa las emisiones de PMIO cuando la actividad económica de sus vecinos se incrementa.

TABLa 2 Estimación de los contaminantes y las pyMEs con datos de panel y modelo Durbin, 1999-2009

\begin{tabular}{|c|c|c|c|c|c|c|c|c|}
\hline \multirow{2}{*}{ PYMES } & \multicolumn{8}{|c|}{ I) DIÓXIDO DE AZUFRE ( $\mathrm{sO}_{2}$ ) } \\
\hline & \multicolumn{4}{|c|}{ DATOS DE PANEL } & \multicolumn{4}{|c|}{ MODELO DURBIN } \\
\hline \multirow[b]{2}{*}{ VARIABLES } & A) & в) & c) & D) & E) & F) & G) & H) \\
\hline & ORIGINAL & CAPITAL & $\begin{array}{l}\text { UNIDAD } \\
\text { ECONÓ- } \\
\text { MICA }\end{array}$ & TOTAL & ORIGINAL & CAPITAL & $\begin{array}{c}\text { UNIDAD } \\
\text { ECONÓ- } \\
\text { MICA }\end{array}$ & TOTAL \\
\hline \multirow{2}{*}{ va } & $1,371^{* * *}$ & $1,371^{* * *}$ & $1,396^{* * *}$ & $1,436^{* * *}$ & 0,74 & 0,786 & $0,971^{*}$ & $1,207^{* *}$ \\
\hline & $(3,047)$ & $(2,986)$ & $(3,143)$ & $(3,167)$ & $(1,318)$ & $(1,347)$ & $(1,711)$ & $(2,049)$ \\
\hline \multirow{2}{*}{ va2 } & $0,087^{*}$ & $0,087^{*}$ & $0,095 * *$ & $0,101^{* *}$ & 0,075 & 0,079 & $0,104^{*}$ & $0,127^{* *}$ \\
\hline & $(1,929)$ & $(1,873)$ & $(2,129)$ & $(2,179)$ & $(1,364)$ & $(1,376)$ & $(1,835)$ & $(2,156)$ \\
\hline \multirow{2}{*}{$\mathrm{k}$} & & $-0,001$ & & 0,101 & & $-0,043$ & & $-0,021$ \\
\hline & & $(-0,005)$ & & $(0,517)$ & & $(-0,232)$ & & $(-0,113)$ \\
\hline \multirow{2}{*}{ ue } & & & $-0,263$ & $-0,29^{*}$ & & & $-0,652^{*}$ & $-0,811^{* *}$ \\
\hline & & & $(-1,642)$ & $(-1,712)$ & & & $(-1,915)$ & $(-2,291)$ \\
\hline \multirow{2}{*}{ constante } & 0,162 & 0,162 & $-0,715$ & $-0,829$ & 1,099 & 0,782 & 0,569 & $-1,04$ \\
\hline & $(0,148)$ & $(0,147)$ & $(-0,592)$ & $(-0,672)$ & $(0,594)$ & $(0,398)$ & $(0,3)$ & $(-0,48)$ \\
\hline \multirow{2}{*}{$\mathrm{w}^{*} \mathrm{va}$} & & & & & 0,955 & 0,851 & 0,685 & 0,312 \\
\hline & & & & & $(0,975)$ & $(0,841)$ & $(0,7)$ & $(0,312)$ \\
\hline \multirow{2}{*}{$\mathrm{w}^{*} \mathrm{va} 2$} & & & & & 0,037 & 0,03 & 0,007 & $-0,015$ \\
\hline & & & & & $(0,374)$ & $(0,294)$ & $(0,077)$ & $(0,156)$ \\
\hline \multirow{2}{*}{$\mathrm{w}^{*} \mathrm{k}$} & & & & & & 0,195 & & 0,613 \\
\hline & & & & & & $(0,58)$ & & $(1,584)$ \\
\hline \multirow{2}{*}{$w^{*}$ ue } & & & & & & & 0,527 & 0,522 \\
\hline & & & & & & & $(1,463)$ & $(1,411)$ \\
\hline
\end{tabular}

\begin{tabular}{|l|c|c|c|c|c|c|c|c|}
\hline \multirow{3}{*}{ PYMES } & \multicolumn{7}{|c|}{ II) PARTÍCULAS FINAS (PMIO) } \\
\cline { 2 - 10 } & \multicolumn{7}{|c|}{ DATOS DE PANEL } & \multicolumn{3}{c|}{ MODELO DURBIN } \\
\hline \multirow{4}{*}{ VARIABLES } & A) & B) & C) & D) & E) & F) & G) & H) \\
\cline { 2 - 10 } & ORIGINAL & CAPITAL & $\begin{array}{c}\text { UNIDAD } \\
\text { ECONÓ- } \\
\text { MICA }\end{array}$ & TOTAL & ORIGINAL & CAPITAL & $\begin{array}{c}\text { UNIDAD } \\
\text { ECONó- } \\
\text { MICA }\end{array}$ & TOTAL \\
\hline \multirow{3}{*}{ va } & 0,237 & 0,163 & 0,248 & 0,179 & $-0,451$ & $-0,647$ & $-0,438$ & $-0,673$ \\
\cline { 2 - 10 } & $(0,609)$ & $(0,415)$ & $(0,635)$ & $(0,45)$ & $(-0,906)$ & $(-1,282)$ & $(-0,847)$ & $(-1,256)$ \\
\hline \multirow{3}{*}{ va2 } & 0,004 & $-0,005$ & 0,007 & $-0,002$ & $-0,056$ & $-0,077$ & $-0,055$ & $-0,079$ \\
\cline { 2 - 10 } & $(0,102)$ & $(-0,146)$ & $(0,194)$ & $(-0,06)$ & $(-1,155)$ & $(-1,551)$ & $(-1,065)$ & $(-1,485)$ \\
\hline \multirow{2}{*}{$\mathrm{k}$} & & $-0,199$ & & $-0,174$ & & $-0,171$ & & $-0,192$ \\
\cline { 2 - 9 } & & $(-1,227)$ & & $(-1,019)$ & & $(-1,058)$ & & $(-1,106)$ \\
\hline
\end{tabular}


(continuación)

\begin{tabular}{|c|c|c|c|c|c|c|c|c|}
\hline \multirow{2}{*}{ PYMES } & \multicolumn{8}{|c|}{ II) PARTÍCULAS FINAS (PMIO) } \\
\hline & \multicolumn{4}{|c|}{ DATOS DE PANEL } & \multicolumn{4}{|c|}{ MODELO DURBIN } \\
\hline & A) & в) & c) & D) & E) & F) & G) & H) \\
\hline VARIABLES & ORIGINAL & CAPITAL & $\begin{array}{c}\text { UNIDAD } \\
\text { ECONÓ- } \\
\text { MICA }\end{array}$ & TOTAL & ORIGINAL & CAPITAL & $\begin{array}{c}\text { UNIDAD } \\
\text { ECONÓ- } \\
\text { MICA }\end{array}$ & TOTAL \\
\hline \multirow{2}{*}{ ue } & & & $-0,115$ & $-0,069$ & & & $-0,073$ & 0,008 \\
\hline & & & $(-0,815)$ & $(-0,465)$ & & & $(-0,235)$ & $(0,026)$ \\
\hline \multirow[t]{2}{*}{ constante } & $-0,805$ & $-0,757$ & $-1,189$ & $-0,993$ & 1,985 & 2,682 & 1,626 & 3,039 \\
\hline & $(-0,847)$ & $(-0,799)$ & $(-1,119)$ & $(-0,92)$ & $(1,211)$ & $(1,581)$ & $(0,941)$ & $(1,54)$ \\
\hline \multirow[t]{2}{*}{$\mathrm{w}^{*} \mathrm{va}$} & & & & & $1,858^{* *}$ & $2,098^{* *}$ & $1,861 * *$ & $2,12^{* *}$ \\
\hline & & & & & $(2,141)$ & $(2,397)$ & $(2,087)$ & $(2,325)$ \\
\hline \multirow[t]{2}{*}{$\mathrm{w}^{*} \mathrm{va} 2$} & & & & & $0,177 * *$ & $0,195^{* *}$ & $0,18^{*}$ & $0,194^{* *}$ \\
\hline & & & & & $(2)$ & $(2,202)$ & $(1,962)$ & $(2,103)$ \\
\hline \multirow[t]{2}{*}{$\mathrm{w}^{*} \mathrm{k}$} & & & & & & $-0,343$ & & $-0,409$ \\
\hline & & & & & & $(-1,177)$ & & $(-1,162)$ \\
\hline \multirow[t]{2}{*}{$\mathrm{w}^{*}$ ue } & & & & & & & $-0,036$ & 0,064 \\
\hline & & & & & & & $(-0,11)$ & $(0,19)$ \\
\hline
\end{tabular}

FUENTE ELABORACIÓN PROPIA CON BASE EN INFORMACIÓN DE LOS CENSOS ECONÓMICOS Y DEL SEMARNAT DE I 999, 2004 Y 2009. ***, **,: SON LOS NIVELES DE SIGNIFICANCIA DEL I \%, $5 \%$ Y IO\%. EN LOS PARÉNTESIS SE MUESTRAN LAS PROBABILIDADES DE CADA COEFICIENTE ESTIMADO

En la tabla 3 se presenta la estimación de los contaminantes más significativos para las grandes empresas. En el caso del $\mathrm{sO}_{2}$, la producción impacta positiva y significativamente a las emisiones de este contaminante (columnas A, в, с у в). En este caso, el modelo Durbin no es significativo, no hay evidencia de efectos espaciales (columnas E, F, G y H). Para las emisiones de pMio en las grandes empresas, la única variable significativa es el capital, con un coeficiente de alrededor del -0,17 (columnas в у в), lo que indicaría que la mejora tecnológica ha influido en la disminución de ese contaminante. La versión espacial de la ecuación mantiene la significancia del capital en la ecuación con coeficientes muy similares al ya referido; sin embargo, en este caso el número de unidades económicas en los estados vecinos sí tiene un efecto espacial negativo, lo cual implica que el crecimiento de grandes empresas genera algún tipo de externalidad positiva en las emisiones contaminantes de los estados vecinos. 
TABLA 3 Estimación de los contaminantes y las grandes empresas con datos de panel y modelo Durbin, 1999-2009

\begin{tabular}{|c|c|c|c|c|c|c|c|c|}
\hline GRANDES & \multicolumn{8}{|c|}{ I) DIÓXIDO DE AZUFRE ( $\mathrm{sO}_{2}$ ) } \\
\hline \multirow[b]{3}{*}{ VARIABLES } & \multicolumn{4}{|c|}{ DATOS DE PANEL } & \multicolumn{4}{|c|}{ MODELO DURBIN } \\
\hline & A) & в) & c) & D) & E) & $\mathbf{F}$ & G) & н) \\
\hline & ORIGINAL & CAPITAL & $\begin{array}{l}\text { UNIDAD } \\
\text { ECONÓ- } \\
\text { MICA }\end{array}$ & TOTAL & ORIGINAL & CAPITAL & $\begin{array}{l}\text { UNIDAD } \\
\text { ECONÓ- } \\
\text { MICA }\end{array}$ & TOTAL \\
\hline \multirow{2}{*}{$\mathrm{Va}$} & $0,987^{* * *}$ & $0,976^{* * *}$ & $0,872^{* * *}$ & $0,87^{* * *}$ & $0,747^{* * *}$ & $0,712^{* *}$ & $0,634^{* *}$ & $0,599^{* *}$ \\
\hline & $(5,2)$ & $(5,191)$ & $(4,458)$ & $(4,479)$ & $(2,762)$ & $(2,65)$ & $(2,286)$ & $(2,174)$ \\
\hline \multirow{2}{*}{ va2 } & $0,076^{* * *}$ & $0,073^{* * *}$ & $0,065^{* * *}$ & $0,064^{* * *}$ & $0,061 * *$ & $0,059^{* *}$ & $0,051^{* *}$ & $0,049^{* *}$ \\
\hline & $(3,641)$ & $(3,548)$ & $(3,115)$ & $(3,059)$ & $(2,58)$ & $(2,504)$ & $(2,128)$ & $(2,065)$ \\
\hline \multirow{2}{*}{$\mathrm{k}$} & & $-0,139$ & & $-0,125$ & & $-0,178^{*}$ & & $-0,17^{*}$ \\
\hline & & $(-1,525)$ & & $(-1,385)$ & & $(-1,757)$ & & $(-1,69)$ \\
\hline \multirow{2}{*}{ ue } & & & $-16,545 *$ & $-15,442 *$ & & & $-16,119^{*}$ & $-16,226^{*}$ \\
\hline & & & $(-1,88)$ & $(-1,76)$ & & & $(-1,719)$ & $(-1,739)$ \\
\hline \multirow[t]{2}{*}{ constante } & $-1,733^{* * *}$ & $-1,55^{* * *}$ & $-1,869^{* * *}$ & $-1,695^{* * *}$ & $-1,384 * *$ & $-1,324 * *$ & $-1,548 * *$ & $-1,499^{* *}$ \\
\hline & $(-4,337)$ & $(-3,748)$ & $(-4,691)$ & $(-4,086)$ & $(-2,371)$ & $(2,27)$ & $(-2,651)$ & $(-2,559)$ \\
\hline \multirow[t]{2}{*}{$\mathrm{w}^{*} \mathrm{va}$} & & & & & 0,415 & 0,432 & 0,394 & 0,434 \\
\hline & & & & & $(1,233)$ & $(1,27)$ & $(1,155)$ & $(1,263)$ \\
\hline \multirow[t]{2}{*}{$\mathrm{w}^{*} \mathrm{va2}$} & & & & & 0,033 & 0,033 & 0,031 & 0,033 \\
\hline & & & & & $(0,948)$ & $(0,914)$ & $(0,879)$ & $(0,921)$ \\
\hline \multirow[t]{2}{*}{$\mathrm{w}^{*} \mathrm{k}$} & & & & & & 0,113 & & 0,136 \\
\hline & & & & & & $(0,847)$ & & $(1,025)$ \\
\hline \multirow[t]{2}{*}{$\mathrm{w}^{*}$ ue } & & & & & & & $-1,425$ & $-0,335$ \\
\hline & & & & & & & $(-0,142)$ & $(-0,033)$ \\
\hline
\end{tabular}

\begin{tabular}{|c|c|c|c|c|c|c|c|c|}
\hline GRANDES & \multicolumn{8}{|c|}{ II) PARTÍCULAS FINAS (PMIO) } \\
\hline \multirow[b]{3}{*}{ VARIABLES } & \multicolumn{4}{|c|}{ DATOS DE PANEL } & \multicolumn{4}{|c|}{ MODELO DURBIN } \\
\hline & A) & B) & c) & D) & E) & F) & G) & H) \\
\hline & ORIGINAL & CAPITAL & $\begin{array}{c}\text { UNIDAD } \\
\text { ECONÓ- } \\
\text { MICA }\end{array}$ & TOTAL & ORIGINAL & CAPITAL & $\begin{array}{c}\text { UNIDAD } \\
\text { ECONÓ- } \\
\text { MICA }\end{array}$ & TOTAL \\
\hline \multirow{2}{*}{ va } & 0,182 & 0,168 & 0,158 & 0,154 & $-0,204$ & $-0,245$ & $-0,127$ & $-0,159$ \\
\hline & $(1,057)$ & $(1,002)$ & $(0,866)$ & $(0,87)$ & $(-0,857)$ & $(-1,061)$ & $(-0,547)$ & $(-0,7)$ \\
\hline \multirow{2}{*}{ va2 } & 0,008 & 0,005 & 0,006 & 0,003 & $-0,012$ & $-0,016$ & $-0,008$ & $-0,011$ \\
\hline & $(0,433)$ & $(0,275)$ & $(0,308)$ & $(0,204)$ & $(-0,592)$ & $(-0,811)$ & $(-0,441)$ & $(-0,598)$ \\
\hline \multirow{2}{*}{$\mathrm{k}$} & & $-0,174^{* *}$ & & $-0,172^{* *}$ & & $-0,177^{* *}$ & & $-0,155^{*}$ \\
\hline & & $(-2,137)$ & & $(-2,09)$ & & $(-2,025)$ & & $(-1,87)$ \\
\hline \multirow{2}{*}{ ue } & & & $-3,483$ & $-1,962$ & & & 4,096 & 5,123 \\
\hline & & & $(-0,424)$ & $(-0,244)$ & & & $(0,521)$ & $(0,665)$ \\
\hline \multirow[t]{2}{*}{ constante } & $-1,257^{* * *}$ & $-1,027^{* * *}$ & $-1,285^{* * *}$ & $-1,045^{* * *}$ & $-1,085^{* *}$ & $-0,934 *$ & $-1,213^{* *}$ & $-1,062 * *$ \\
\hline & $(-3,464)$ & $(-2,784)$ & $(-3,461)$ & $(-2,756)$ & $(-2,105)$ & $(-1,859)$ & $(-2,477)$ & $(-2,197)$ \\
\hline
\end{tabular}


(continuación)

\begin{tabular}{|c|c|c|c|c|c|c|c|c|}
\hline GRANDES & \multicolumn{8}{|c|}{ II) PARTÍCULAS FINAS (PMIO) } \\
\hline \multirow[b]{3}{*}{ VARIABLES } & \multicolumn{4}{|c|}{ DATOS DE PANEL } & \multicolumn{4}{|c|}{ MODELO DURBIN } \\
\hline & A) & в) & c) & D) & E) & F) & G) & н) \\
\hline & ORIGINAL & CAPITAL & $\begin{array}{c}\text { UNIDAD } \\
\text { ECONÓ- } \\
\text { MICA }\end{array}$ & TOTAL & ORIGINAL & CAPITAL & $\begin{array}{l}\text { UNIDAD } \\
\text { ECONÓ- } \\
\text { MICA }\end{array}$ & TOTAL \\
\hline \multirow[t]{2}{*}{$\mathrm{w}^{*} \mathrm{va}$} & & & & & 0,445 & 0,367 & 0,241 & 0,195 \\
\hline & & & & & $(1,497)$ & $(1,253)$ & $(0,844)$ & $(0,691)$ \\
\hline \multirow{2}{*}{$\mathrm{w}^{*} \mathrm{va2}$} & & & & & 0,023 & 0,01 & 0,009 & 0 \\
\hline & & & & & $(0,736)$ & $(0,321)$ & $(0,314)$ & $(-0,005)$ \\
\hline \multirow[t]{2}{*}{$\mathrm{w}^{*} \mathrm{k}$} & & & & & & $-0,062$ & & $-0,045$ \\
\hline & & & & & & $(-0,542)$ & & $(-0,415)$ \\
\hline \multirow[t]{2}{*}{$\mathrm{w}^{*} \mathrm{ue}$} & & & & & & & $-26,438^{* * *}$ & $-24,282^{* * *}$ \\
\hline & & & & & & & $(-3,153)$ & $(-2,948)$ \\
\hline
\end{tabular}

FUENTE ELABORACIÓN PROPIA CON BASE EN INFORMACIÓN DE LOS CENSOS ECONÓMICOS Y DEL SEMARNAT DE I999, 2004 Y 2009. ${ }^{* * *},{ }^{* *}, *$ : SON LOS NIVELES DE SIGNIFICANCIA DEL I\%, $5 \%$ Y IO\%. EN LOS PARÉNTESIS SE MUESTRAN LOS ESTADÍSTICOS T DE CADA COEFICIENTE ESTIMADO

\section{Conclusiones}

En este trabajo se analizaron cuatro diferentes tipos de contaminantes para diferentes tamaños de empresa en los estados mexicanos. No fue posible confirmar para todos los casos la existencia general de una curva medioambiental de Kuznets en el sentido tradicional. En los diferentes tamaños de empresa se encontró evidencia de que hay emisiones contaminantes que son impactadas positivamente, ya sea por la producción o por el número de empresas que participan en la industria manufacturera. Solo en el caso de las grandes empresas fue posible identificar que el cambio tecnológico opera a favor de la disminución de los contaminantes.

Las microempresas tienen un efecto significativo en la generación de emisiones de co y pmio. En el primer caso, la producción de las microempresas no tiene efectos relevantes directos en la generación de emisiones y parecería operar más bien una especie de externalidad positiva, que ocurre al aumentar el número de microempresas en las vecindades de algún estado en particular. Para el PMio hay efectos directos que se generan al elevarse la producción de estas empresas en los estados del país; sin embargo, opera también la externalidad espacial positiva, por el crecimiento de unidades económicas en la vecindad de los estados.

$\mathrm{Al}$ considerar las estimaciones para las PYMEs, solo resultó significativo su impacto en las emisiones de $\mathrm{SO}_{2}$ y PM Io. En el primer caso fue posible confirmar un efecto positivo del valor agregado y su cuadrado en la generación de emisiones, sin que hubiera evidencia alguna de efectos espaciales o externalidades generadas por las empresas de los estados vecinos. Para las pmio no se verificaron efectos directos de estas empresas localizadas en los estados, pero sí de las empresas localizadas en sus vecindades, lo cual es indicativo nuevamente de una externalidad, aunque negativa, ya que la operación de empresas vecinas eleva la generación de emisiones de $\mathrm{SO}_{2} \mathrm{y}$ PMIO. 
Finalmente, para el caso de las grandes empresas, los resultados indican que su actividad impacta significativamente en las emisiones de $\mathrm{SO}_{2}$ у рмго. En particular para el $\mathrm{SO}_{2}$, se encontró evidencia de que la producción de ese tipo de empresas en una entidad federativa eleva las cantidades per cápita de ese contaminante, sin existir ningún tipo de efecto espacial por la operación de grandes empresas en los estados vecinos. Caso contrario es el de las emisiones de pmio, en las cuales se verifica un efecto tecnológico positivo, ya que los procesos de inversión en esas empresas tienden a reducir sus emisiones contaminantes. Al mismo tiempo, para las grandes empresas, existe evidencia de un efecto positivo espacial en relación con el aumento del número de empresas de ese mismo tamaño en estados vecinos. La ventaja de usar el panel espacial es que permite determinar los impactos directos e indirectos que existen sobre la contaminación. En este caso, lo que nos dice es que el incremento de las unidades económicas de las grandes empresas al interior de una región tiende a influir en la disminución de PM Io en las demás regiones.

Finalmente, el uso de modelos tipo Kuznets por tamaño de empresa permite plantear que no es posible realizar estimaciones generales como las que hasta la fecha se han realizado sobre este tema para México, ya que existe un efecto diferenciado del tamańo empresarial en el tipo de contaminante en consideración, por lo cual no es posible realizar generalizaciones. También es relevante tomar en cuenta la existencia de externalidades espaciales, las cuales tienden a profundizar los problemas de la contaminación o a atenuarlos según sea el tipo de empresa que se considera. Siguen siendo aún limitados los datos sobre emisiones contaminantes por regiones, por lo cual es necesario seguir profundizando en el tema y, en la medida en que se cuenta con mayor información, utilizar muestras más grandes para obtener resultados más robustos.

\section{Referencias bibliográficas}

Anselin, L. (1988). Spatial econometrics: Methods and model. Boston: Kluwer Academic Publishers.

Anselin, L. \& Florax, R. (1995). New directions in spatial econometrics. Nueva York: SpringerVerlag.

Anselin, L., LeGayo, J. \& Jayet, H. (2008). Spatial panel econometrics. En L. Mátyás \& P. Sevestre, P. (eds.), The econometrics of panel data. Berlín / Heidelberg: Springer-Verlag.

Antle, J. M. \&Heidebrink, G. (1995). Environment and development: Theory and international evidence. Economic Development and Cultural Change, 43(3), 603-625. http://doi. org/10.1086/452171

Antweiler, W., Copeland, B. \& Taylor, M. S. (2001). Is free trade good for the environment? American Economic Review, 91(4), 877-908. http://doi.org/10.3386/w6707

Arbia, G. (2008). Spatial econometrics: Statistical foundations and applications to regional convergence (Advances in Spatial Science). Berlín: Springer.

Baltagi, B. H. (2005). Econometric analysis of panel data ( $3^{\mathrm{a}} \mathrm{ed}$.). Oxford, uk: John Wiley and Sons. 
Bartik, T. (1988). The effects of environmental regulation on business location in the United States. Growth and Change, 19(3), 22-44. http://doi.org/10.1111/j.1468-2257.1988. tb00473.x

Birdsall, N. \& Wheeler, D. (1993). Trade policy and industrial pollution in Latin America: Where are the pollution havens? Journal of Environment and Development, 2(1), 137149. En http://infohouse.p2ric.org/ref/22/21762.pdf

Boopen, S. \& Vinesh, S. (2010). On the relationship between $\mathrm{CO} 2$ emissions and economic growth: The Mauritian experience. En http://www.dl.icdst.org/pdfs/files/35983d7b0f 9ae1d0d649a5c96b267272.pdf

Carson, R. T. (2010). The Environmental Kuznets Curve: Seeking empirical regularity and theoretical structure. Review of Environmental Economics and Policy, 4(1), 3-23. https:// doi.org/10.1093/reep/rep021

Cliff, D. \& Ord, K. (1972). Testing for spatial autocorrelation among regression residuals. Geographical Analysis, 4(3), 267-284. http://doi.org/10.1111/j.1538-4632.1972. tb00475.x

Cole, M. (2003). Development, trade, and the environment: How robust is the Environmental Kuznets Curve? Environment and Development Economics, 8(4), 557-580. https://doi. org/10.1017/S1355770X0300305

Cole, M., Elliott, R. J. R. \& Shimamoto, K. (2005). Industrial characteristics, environmental regulations and air pollution: An analysis of the UK manufacturing sector. Journal of Environmental Economics and Management, 50(1), 121-143. https://doi.org/10.1016/j. jeem.2004.08.001

Comisión Económica para América Latina y el Caribe (CEPAL). (2004). Cooperación ambiental en el TLCAN y perspectivas para el TLCCA-EeUu [LC/MEX/R.866]. México, D.F.: Cepal. En http://repositorio.cepal.org/bitstream/handle/11362/25732/1/LCMEXR866s_es.pdf

Cravo, T., Gourlay, A. \& Becker, B. (2012). sMes and regional economic growth in Brazil. Small Business Economics, 38(2), 217-230. https://dx.doi.org/10.1007/s11187-010-9261-z

Dasgupta, S., Lucas, R. E. B. \& Wheeler, D. (2002). Plant size, industrial air pollution, and local income: evidence from Mexico and Brazil. Environment and Development Economics, 7(2), 365-381. https://doi.org/10.1017/S1355770X02000220

Day, K. \& Grafton, R. Q. (2001). Economic growth and the environment: A Canadian perspective. Ottawa: University of Ottawa.

Díaz-Vázquez, M. (2009). The dissociation between emissions and economic growth: The role of shocks exogenous to the Environmental Kuznets Curve model. Applied Econometrics and International Development, 9(2). En http://www.usc.es/economet/reviews/ aeid923.pdf

Díaz-Vázquez, M. \& Cancelo, M. T. (2009). Emisiones de Co2 y azufre y crecimiento económico: ¿Una curva de Kuznets ambiental? Regional and Sectoral Economic Studies, 9(2). En http://www.usc.es/economet/reviews/eers927.pdf

Dinda, S. (2004). Environmental Kuznets Curve hypothesis: A survey. Ecological Economics, 49(4), 431-455. http://doi.org/10.1016/j.ecolecon.2004.02.011

Ederington, J., Levinson, A. \& Minier, J. (2004). Trade liberalization and pollution havens. Advances in Economic Analysis and Policy, 4, 215-247. http://doi.org/10.3386/w10585 
Elhorst, J. P. (2003). Unconditional maximum likelihood estimation of dynamic models for spatial panels. Research Report 03C27. University of Groningen. En http://www.rug. $\mathrm{nl} /$ research/portal/files/3015787/03c27.pdf

Elhorst, J. P. (2010). Spatial panel data models. En M. Manfred \& A. G. Fischer (eds.), Handbook of Applied Spatial Analysis. Software tools, methods and applications (pp. 377407). Berlín: Springer-Verlag.

Ertur, C. \& Koch, W. (2007). Growth, technological interdependence and spatial externalities: Theory and evidence. Journal of Applied Econometrics, 22(6), 1033-1062. http://doi. org/10.1002/jae.963

Gallagher, K. (1999). Pollution intensive industry in Mexico under NAFTA: Model and empirical evidence. The Fletcher Journal of Development Studies 1999: Does development work? http://fletcher.tufts.edu/praxis/archives/xv/gallagher.pdf

Gallagher, K. (2000). Trade liberalization and industrial pollution in Mexico: Lessons for the FTAA. Working Paper No. 00-07, Global Development and Environment Institute. En http://econwpa.repec.org/eps/it/papers/0106/0106003.pdf

Gallagher, K. (2004). Free trade and the environment. Mexico, NAFTA and beyond. Stanford, CA: Stanford University Press.

Gallagher, K. (2006). Is NAFTA working for Mexico? The Environmental Forum, 23(3\&4), 21-27.

Grossman, G. M. \& Krueger, A. B. (1991). Environmental impacts of a North American Free Trade Agreement. NBer Working Paper 3914. Cambridge, MA: National Bureau of Economic Research (NBER). http://doi.org/10.3386/w3914

Grossman, G. M. \& Krueger, A. B. (1993). Environmental impacts of a North American Free Trade Agreement. En P. M. Gaber (ed.), The Mexico-U.S. Free Trade Agreement (pp. 13-56). Cambridge, ma: Miт Press.

Harbaugh, W. T., Levinson, A. \& Wilson, D. M. (2002). Reexamining the empirical evidence For an Environmental Kuznets Curve. Nber Working Paper No. 7711. The Review of Economics and Statistics, 84(3), 541-551. http://doi.org/10.3386/w7711

Hertwich, E. G. (2011). The life cycle environmental impacts of consumption. Economic System Research, 23(1), 27-47. http://dx.doi.org/10.1080/09535314.2010.536905

Jiang, L., Chen, L. \& Ping, L. (2014). The determinants of pollution levels: Firm-level evidence from Chinese manufacturing. Journal of Comparative Economics, 42(1), 118-142. https://doi.org/10.1016/j.jce.2013.07.007

Jones, L. E. \& Manuelli, R. E. (2000). Endogenous policy choice: The case of pollution and growth. Staff Report 276, Federal Reserve Bank of Minneapolis. Publicado en Review of Economic Dynamics, 4(2), 369-405. https://doi.org/10.1006/redy.2000.0118

Kochi, I. \& Medina, L. P. C. (2013). Beyond the Environmental Kuznets Curve: Understanding the determinants of environmental degradation in Mexico. Nóesis. Revista de Ciencias Sociales y Humanidades, 22(43), 52-83. En http://www.redalyc.org/ pdf/859/85927874003.pdf

Lesage, J. \& Fischer, M. (2008). Spatial growth regressions: Model specification, estimation and interpretation. Spatial Economic Analysis, 3(3), 275-304. http://dx.doi. org/10.1080/17421770802353758

Lesage, J. \& Pace, R. K. (2009). Introduction to spatial econometrics. Londres: CRC Press / Taylor \& Francis Group. 
Lieb, C. M. (2003). The Environmental Kuznets Curve - A Survey of the empirical evidence and of possible causes. Discussion Paper Series No. 391. University of Heidelberg, Department of Economics. En http://www.uni-heidelberg.de/md/awi/forschung/dp391.pdf

Levinson, A. (1996). Environmental regulations and manufacturers' location choices: Evidence from the Census of Manufactures. Journal of Public Economics, 62(1-2), 5-29. https:// doi.org/10.1016/0047-2727(96)01572-1

Lipford, J. W. \& Yandle, B. (2011). NAFTA, Environmental Kuznets Curves, and Mexico's progress. Working Paper. Bozeman, mT: Property and Environmental Research Center (PERC). En https://www.perc.org/sites/default/files/Kuznets\%20Yandle\%20Lipford.pdf

López, R. (1994). The environment as a factor of production: The effects of economic growth and trade liberalization. Journal of Environmental Economics and Management, 27(2), 163-184. https://doi.org/10.1006/jeem.1994.1032

López-Bazo, E., Vaya, E. \& Artis, M. (2004). Regional externalities and growth: Evidence from European regions. Journal of Regional Science, 44(1), 43-73. https://doi.org/10.1111/ j.1085-9489.2004.00327.x

López-Cordova, E. (2001). NAFTA and the Mexican economy: Analytical issues and lessons for the FTAA, INTAL-ITD-STA. Occasional Paper 9. Washington, D.C.: Instituto par la Integración de América Llatina y el Caribe and Inter-American Development Bank's Integration, Trade, and Hemspherc Issues Division and Statistics and Quantitavive Analysis Unit.

Marshall, C. (1998). Economic instruments and the business use of energy: a consultation paper. Task Force Report, Stationery Office. Londres: Government Task Force on the Industrial Use of Energy. En http://webarchive.nationalarchives.gov.uk/20130129110402/ http:/www.hm-treasury.gov.uk/d/EconomicInstruments.pdf

Merlevedea, B., Verbekeb, T. \& Clercq, M. (2006). The EKC for $\mathrm{so}_{2}$ : Does firm size matter? EcologicalEconomics, 59(4), 451-461. https://doi.org/10.1016/j.ecolecon.2005.11.010

Millo, G. \& Piras, G. (2012). Splm: Spatial Panel Data Models. R. Journal of Statistical Software, 47(1), 1-38. http://dx.doi.org/10.18637/jss.v047.i01

Organisation for Economic Co-operation and Development (OECD). (1994). Methodologies for environmental and trade reviews [OCDE/GD(94) I03]. París: OECD. En http://www.oecd. org/tad/envtrade/36767000.pdf

Paelinck, P. \& Klaassen, H. [with the assistance of J.-P. Ancot, A. C. P. Verster, Sj. Wagenaar]. (1979). Spatial Econometrics. Farnborough, Eng.: Saxon House.

Panayotou, T., Peterson, A. \& Sachs, J. (2000). Is the Environmental Kuznets Curve driven by structural change? What extended time series may imply for developing countries. Cambridge, мA: Harvard Institute for International Development. https://doi. org/10.7916/D8CV4QJF

Parker, C., Redmond, M. J. \& Simpson, M. (2009). A review of interventions to encourage SMEs to make environmental improvements. Environment and Planning C: Government and Policy, 27(2), 279-301.

Qi, J., Tang, X. \& Xi, X. (2015). The size distribution of firms and industrial pollution. Working Paper. En https://sites.google.com/site/zjutangxin/research

Revell, A. \& Blackburn, R. (2007). The business case for sustainability? An examination of small firms in the UK's construction and restaurant sectors. Business Strategy and the Environment, 16(6), 404-420. https://doi.org/10.1002/bse.499 
Revell, A. Stokes, D. \& Chen, H. (2010). Small businesses and the environment: Turning over a new leaf? Business Strategy and the Environment, 19(5), 273-288. https://doi. org $/ 10.1002 /$ bse. 628

Roca, J. (2003). Do individual preferences explain the Environmental Kuznets Curve? Ecological Economics, 45(1), 3-10. https://doi.org/10.1016/S0921-8009(02)00263-X

Roca, J. \& Padilla, E. (2003). Emisiones atmosféricas y crecimiento económico en España: la curva de Kuznets ambiental y el Protocolo de Kyoto. Economía Industrial, (351), 73-86. En http://www.minetad.gob.es/Publicaciones/Publicacionesperiodicas/EconomiaIndustrial/ RevistaEconomiaIndustrial/351/Economia05.pdf

Ruttan, V. (1971). Technology and the environment. American Journal of Agricultural Economics, 53(5), 707-717. https://doi.org/10.2307/1238069

Saravia, A. (2002). La curva medioambiental de Kuznets para América Latina y el Caribe. Documentos de Reflexión Académica No. 23. Cochabamba, Bolivia: Proyecto de Mejoramiento de la Formación en Economía (promec)

Selden, T, M. \& Song, D. (1994). Environmental quality and development: Is there a Kuznets curve for air pollution emissions? Journal of Environmental Economics and Management, 27(2), 147-162. https://doi.org/10.1006/jeem.1994.1031

Stern, D. I. (1998). Progress on the Environmental Kuznets Curve? Environment and Development Economics, 3(2), 173-196. https://doi.org/10.1017/S1355770X98000102

Stern, D. I. (2004). The rise and fall of the Environmental Kuznets Curve. World Development, 32(8), 1419-1439. https://doi.org/10.1016/j.worlddev.2004.03.004

Stern, D. I., Common, M. S. \& Barbier, E. B. (1996,) Economic growth and environmental degradation: The Environmental Kuznets Curve and sustainable development. World Development, 24(7), 1151-1160. https://doi.org/10.1016/0305-750X(96)00032-0

Stern, D. I. \& Common, M. S. (2001). Is there an Environmental Kuznets Curve for sulfur? Journal of Environmental Economics and Management, 41(2), 162-178. https://doi. org/10.1006/jeem.2000.1132

Suri, V. \& Chapman, D. (1998). Economic growth, trade and the energy: Implications for the Environmental Kuznets Curve. Ecological Economics, 25(2), 195-208.

Tarazona, M. (1999). El cambio climático en el desarrollo económico: Revisión de la hipótesis de Kuznets. Desarrollo y Sociedad, 43(3), 173-224. En https://ideas.repec.org/a/ $\mathrm{col} / 000090 / 007452 . \mathrm{html}$

Wooldridge, J. (2002). Econometric analysis of cross section and panel data. Cambridge, MA: MIT Press.

Yandle, B., Bhattarai, M. \& Vijayaghavan, M. (2004). Environmental Kuznets curves: A review of findings, methods, and policy implications. Research Study, 02, 1-38. Bozeman, мт: Property and Environmental Research Center (PERC). En https://www.perc.org/ articles/environmental-kuznets-curves 
*

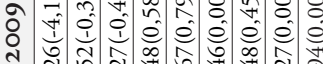

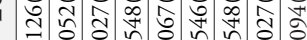

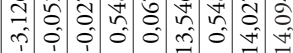

:

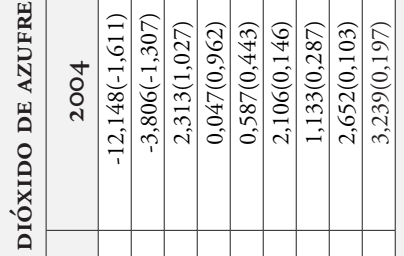

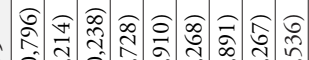

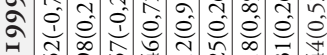

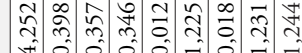

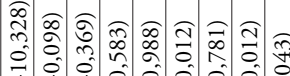

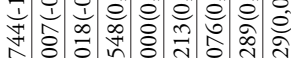

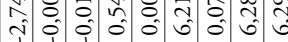

* * *

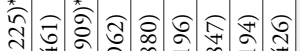

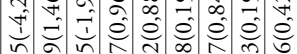

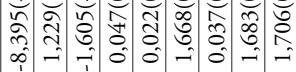

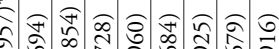

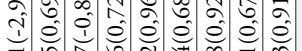
=

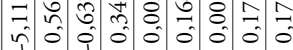

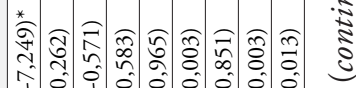

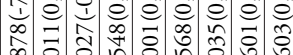

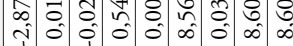

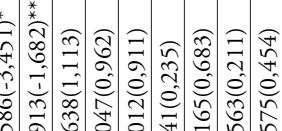

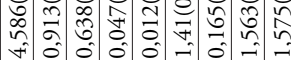

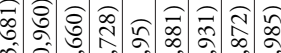
บิ

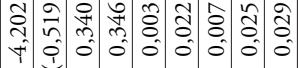

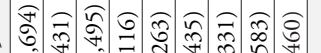

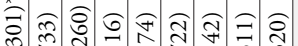

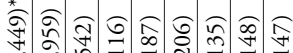
先

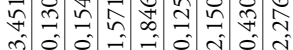

त సิ $\rightarrow 00=0000$

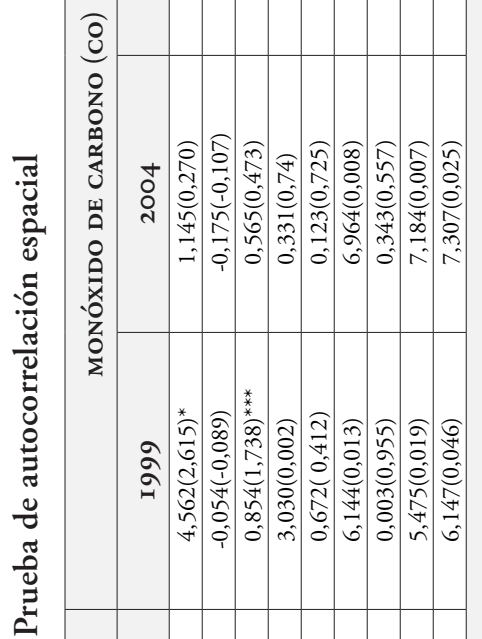

\section{i 0 त}

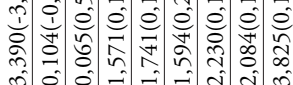

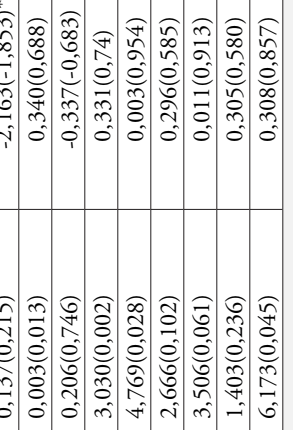

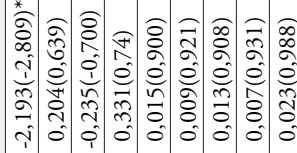

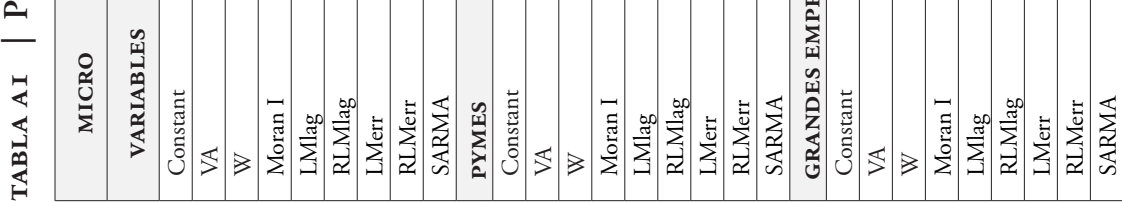




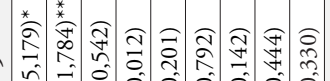

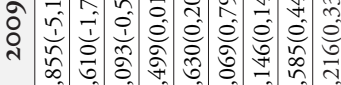

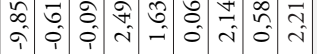

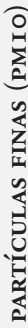

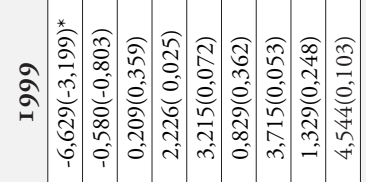

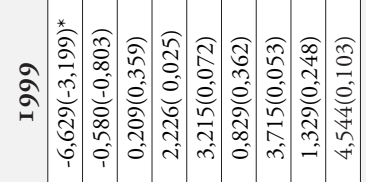

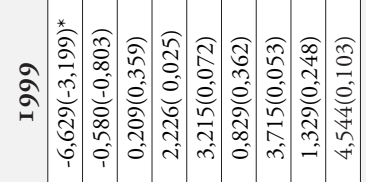

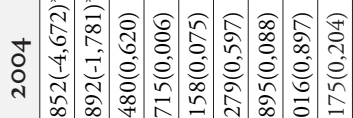

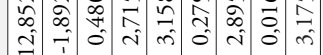

(2)

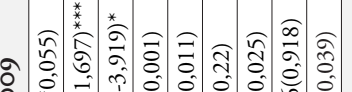

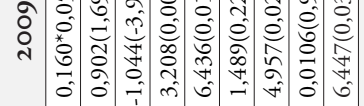

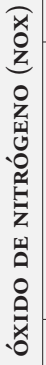

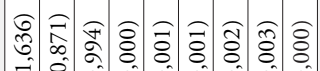

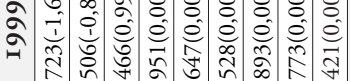

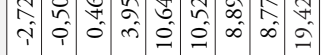

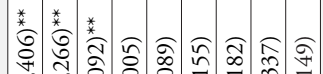

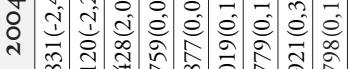

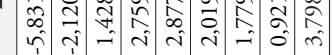

它

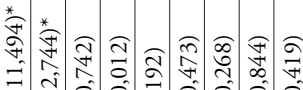

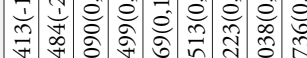

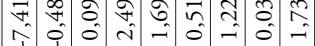

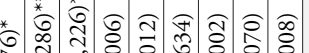

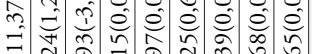

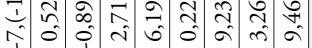

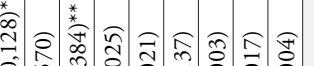

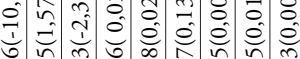

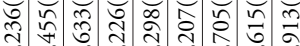

री

\section{\begin{tabular}{ll|l|l|l|}
\hline & -10 & & & -1 \\
\hline
\end{tabular}}

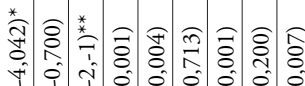

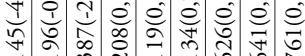

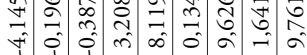




\begin{tabular}{|c|c|c|c|c|c|c|c|c|c|c|}
\hline \multirow{4}{*}{ 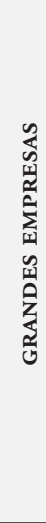 } & है & 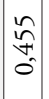 & $\begin{array}{l}\hat{0} \\
\infty \\
0 \\
0\end{array}$ & d & $\begin{array}{l}0 \\
b \\
b \\
b\end{array}$ & $\mid \begin{array}{l}n \\
0 \\
0 \\
0\end{array}$ & $\mid \begin{array}{l}0 \\
\hat{0} \\
\vdots \\
0\end{array}$ & 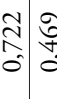 & $\begin{array}{l}0 \\
b \\
b\end{array}$ & 0 \\
\hline & 牙 & 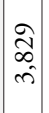 & 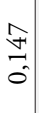 & c & 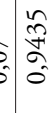 & $\mid \begin{array}{l}\hat{n} \\
\tilde{o} \\
0\end{array}$ & 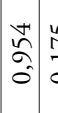 & \begin{tabular}{lll}
$n$ & \multicolumn{1}{c}{} \\
- & $\infty$ \\
0 & &
\end{tabular} & $\begin{array}{ll}0 \\
0 \\
0 \\
0\end{array}$ & 0 \\
\hline & 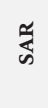 & $\begin{array}{l}\text { సે } \\
\infty \\
\hat{\sim}\end{array}$ & $\begin{array}{l}\stackrel{F}{+} \\
0 \\
0\end{array}$ & c & 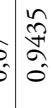 & $\mid \begin{array}{l}\hat{n} \\
\tilde{o} \\
0\end{array}$ & $\begin{array}{l}\stackrel{+}{n} \\
\tilde{\sigma} \\
\tilde{\sigma}\end{array}$ & \begin{tabular}{l|l}
$n$ & $\ddots$ \\
$\vdots$ & 0 \\
0 & 0
\end{tabular} & 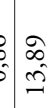 & 0 \\
\hline & 心 & $\left|\begin{array}{c}\widehat{\partial} \\
\infty \\
\tilde{n}\end{array}\right|$ & \begin{tabular}{l}
$\hat{f}$ \\
\multirow{2}{*}{} \\
0
\end{tabular} & c & 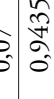 & $\mid \begin{array}{l}\hat{n} \\
\hat{0} \\
0\end{array}$ & 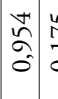 & 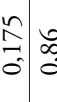 & $\begin{array}{l}0 \\
b \\
0 \\
0\end{array}$ & 0 \\
\hline \multirow{4}{*}{ 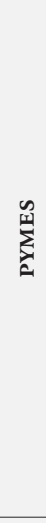 } & के & $\begin{array}{l}\grave{\vdots} \\
\grave{n}\end{array}$ & $\begin{array}{l}+1 \\
0 \\
0 \\
0\end{array}$ & $a$ & 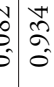 & $\mid \begin{array}{c}2 \\
0 \\
0 \\
0\end{array}$ & $\begin{array}{l}+ \\
0 \\
0\end{array}$ & 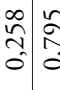 & 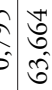 & 0 \\
\hline & س્ద & $\mid \begin{array}{c}\hat{n} \\
\hat{2} \\
\hat{\jmath} \\
\mid\end{array}$ & 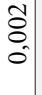 & s & $\begin{array}{c}0 \\
0 \\
0 \\
0\end{array}$ & $\mid \begin{array}{l}0 \\
0 \\
0 \\
0\end{array}$ & $\begin{array}{ll}\infty & 3 \\
\tilde{2} & 3 \\
0 & 3\end{array}$ & 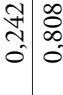 & 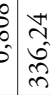 & 0 \\
\hline & $\frac{n}{4}$ & $\begin{array}{l}n \\
\hat{n} \\
\tilde{n} \\
\tilde{n}\end{array}$ & $\begin{array}{l}\tilde{\delta} \\
\text { oे } \\
0\end{array}$ & to & $\begin{array}{ll}0 \\
0 \\
0 \\
0\end{array}$ & $\left|\begin{array}{l}0 \\
0 \\
0 \\
0\end{array}\right|$ & $\begin{array}{c}\infty \\
\tilde{2} \\
\tilde{0}\end{array}$ & 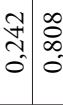 & 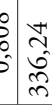 & 0 \\
\hline & 䒺 & $\begin{array}{c}\tilde{n} \\
\hat{n} \\
\tilde{y}\end{array}$ & $\begin{array}{l}\text { है } \\
\text { ठे } \\
0\end{array}$ & to & $\begin{array}{ll}0 \\
0 \\
0 \\
0\end{array}$ & $\mid \begin{array}{l}0 \\
0 \\
0 \\
0\end{array}$ & 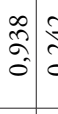 & \begin{tabular}{l|l}
\multirow{I}{f}{} & $\infty$ \\
& $\infty$ \\
0 & 0 \\
0
\end{tabular} & 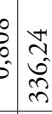 & 0 \\
\hline \multirow{4}{*}{ 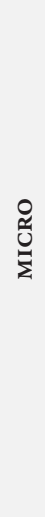 } & के & $\mid \begin{array}{l}\overrightarrow{\hat{b}} \\
\hat{n}\end{array}$ & 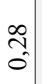 & $\stackrel{0}{0}$ & $\begin{array}{ll}: \\
:\end{array}$ & $\left|\begin{array}{l}n \\
\hat{0} \\
0\end{array}\right|$ & \begin{tabular}{ll}
$\hat{\sigma}$ & \\
$\hat{0}$ & \multicolumn{1}{c}{}
\end{tabular} & \begin{tabular}{l|l}
$\vec{\sim}$ & $\infty$ \\
$\overrightarrow{0}$ & 0
\end{tabular} & 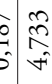 & $\frac{n}{m} a^{2}$ \\
\hline & 眗 & $\stackrel{\hat{\approx}}{\stackrel{2}{\sim}}$ & $\begin{array}{l}\hat{0} \\
+ \\
0 \\
0\end{array}$ & బ & 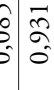 & $\left|\begin{array}{l}\hat{A} \\
\hat{0} \\
0\end{array}\right|$ & $\begin{array}{c}\infty \\
\tilde{\sigma} \\
\tilde{0}\end{array}$ & \begin{tabular}{ll}
\multirow{2}{*}{} \\
\multirow{-}{*}{} \\
-
\end{tabular} & S & to \\
\hline & $\frac{\pi}{4}$ & {$\left[\begin{array}{c}\hat{\tilde{z}} \\
\hat{\sim}\end{array}\right.$} & \begin{tabular}{l}
$\widetilde{O}$ \\
\multirow{+}{0}{} \\
0
\end{tabular} & $\ddot{\infty}$ & \begin{tabular}{c|c}
$\hat{\delta}$ & $\bar{m}$ \\
$\delta^{-}$ & $\hat{\sigma}$
\end{tabular} & $\left|\begin{array}{l}\hat{A} \\
\hat{0} \\
0\end{array}\right|$ & $\begin{array}{c}\infty \\
\tilde{\sigma} \\
\tilde{\sigma}\end{array}$ & \begin{tabular}{ll}
\multirow{2}{*}{} & \multirow{2}{*}{} \\
- & 0 \\
\end{tabular} & to & o \\
\hline & 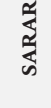 & $\begin{array}{l}\hat{\tilde{n}} \\
\hat{\sim}\end{array}$ & \begin{tabular}{l} 
Ố \\
\multirow{1}{*}{} \\
0
\end{tabular} & $\mathscr{\infty}$ & $\begin{array}{cc}\hat{\delta} & \bar{\Omega} \\
\tilde{\delta} & \tilde{\sigma}\end{array}$ & $\mid \begin{array}{l}\hat{A} \\
0 \\
0\end{array}$ & $\begin{array}{lll}\infty & 1 \\
2 & 1 \\
0 & 1\end{array}$ & 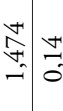 & $\begin{array}{l}b \\
b \\
\mathbb{f}\end{array}$ & b \\
\hline 8 & 奈 & & & 全 & & \multicolumn{2}{|c|}{$\sum_{n}^{J}$} & 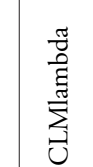 & \multicolumn{2}{|c|}{ 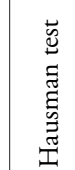 } \\
\hline
\end{tabular}


